\title{
Perinatal Outcomes After Liver Transplantation - Is There A Role For Aspirin Treatment?
}

\section{Gil Zeevi ( $\square$ zeevi07@gmail.com )}

Helen Schneider Hospital for Women, Rabin Medical Center

\section{Marius Braun}

Tel Aviv University

\section{Eviatar Nesher}

Tel Aviv University

\section{Arnon Wiznitzer}

Helen Schneider Hospital for Women, Rabin Medical Center

\section{Eran Hadar}

Helen Schneider Hospital for Women, Rabin Medical Center

\section{Alyssa Hochberg}

Helen Schneider Hospital for Women, Rabin Medical Center

\section{Research Article}

Keywords: Liver transplantation, Aspirin, Pre-eclampsia, Obstetric outcome

Posted Date: October 20th, 2021

DOl: https://doi.org/10.21203/rs.3.rs-940083/v1

License: (c) (i) This work is licensed under a Creative Commons Attribution 4.0 International License.

Read Full License 


\section{Perinatal Outcomes After Liver Transplantation - Is There a Role for Aspirin}

Treatment?

Gil Zeevi ${ }^{1,2}$, MD; Marius Braun, MD ${ }^{2,3}$; Eviatar Nesher ${ }^{2,4}$; Arnon Wiznitzer ${ }^{1,2}$ MD; Eran Hadar $^{1,2}$ MD; Alyssa Hochberg ${ }^{1,2}$, MD,MPH

${ }^{1}$ Helen Schneider Hospital for Women, Rabin Medical Center, Petach Tikva, Israel

${ }^{2}$ Sackler Faculty of Medicine, Tel Aviv University, Tel Aviv, Israel

${ }^{3}$ Liver Institute, Rabin Medical Center, Petach Tikva, Israel

${ }^{4}$ Department of Organ Transplantation, Rabin Medical Center, Petach Tikva, Israel

\section{Correspondence:}

Gil Zeevi, MD

Helen Schneider Hospital for Women, Rabin Medical Center

39 Jabotinsky St. Petach Tikva, Israel. 4941492

Tel: $+972-3-9377680$

Fax: $+972-3-9377683$

Email: zeevi07@gmail.com

Funding: This research did not receive any specific grant from funding agencies in the public, commercial, or not-for-profit sectors.

Disclosure: The authors report no conflict of interest. 


\section{$\underline{\text { Abstract }}$}

Purpose: To describe perinatal outcomes in liver transplanted women and evaluate the effect of low-dose aspirin treatment in these women.

Methods: An observational retrospective study examining perinatal outcomes among 10 liver transplant recipients, between 2016-2020 at a single university-affiliated tertiary medical center in Israel. Demographic, obstetric and clinical data were obtained from electronic health records. Low-dose aspirin use in these women during pregnancy began in 2018 and was evaluated with regards to its effect on the risk of developing preeclampsia and hypertensive disease.

Results: Overall, 13 deliveries in 10 pregnant liver transplant recipients were identified. Primary liver disease was Wilson's in 7 pregnancies (53\%), acute liver failure in $3(23 \%)$, and a single woman with each of the following: Viral hepatitis A, Hyperoxaluria type 1 and primary sclerosing cholangitis. Median age was 23 years at transplant and 30 at conception. All recipients received tacrolimus, steroids were administered to 9 (69\%), and low-dose aspirin (100mg daily) administered in 5 (38\%) pregnancies. Regarding maternal outcomes, 2 women (16\%) developed preeclampsia, $1(8 \%)$ developed gestational hypertension, and $3(24 \%)$ had postpartum infection. Of women receiving low-dose aspirin, none developed hypertensive disease or suffered excessive bleeding. Median gestational age at delivery was 37 weeks (3139 weeks), with 6 preterm births (31-36) and a median birthweight of 3072g (1450-4100g).

Conclusions: Liver-transplanted pregnant women comprise a unique and complex patient population. Based on our single-center experience and due to its potential benefit, low-dose aspirin may by a possible preventive measure for preeclampsia. Further prospective, large studies are needed to delineate specific complications in this patient population and to evaluate treatment options, in order to improve maternal and neonatal outcomes.

Key words: Liver transplantation; Aspirin; Pre-eclampsia; Obstetric outcome. 


\section{Introduction}

During the past few years, an increase in the number of pregnancies achieved following liver transplantation has been observed. These pregnancies are at increased risk for obstetric and neonatal complications (1). Maternal complications include hypertensive disorders of pregnancy, gestational diabetes, post-partum haemorrhage, blood product transfusions, graft rejection, and a higher rate of cesarean deliveries (2). Neonatal complications include preterm birth, intrauterine growth restriction and fetal distress, with no known increase in the incidence of congenital anomalies $(1,3)$.

Since the first case of a pregnancy following a liver transplant over 40 years ago was described (8), major progress has been made in the fields of transplantation and obstetrics. This may be attributed to new surgical techniques, better anesthetic care and improved immunologic and pharmacological treatments, and yet, there is no consensus regarding optimal treatment and surveillance during pregnancy for this unique population. The obstetric field has also seen great progress in the prevention of pre-eclampsia in high-risk patients, including kidney transplant recipients, through early treatment with low-dose aspirin between 12-16 weeks of gestation (4$6)$.

Our tertiary university-affiliated medical center is the largest solid organ transplant center in Israel, centralizing the care of more than 300 transplant recipients each year, with performance of 70 liver transplants annually. Obstetric follow-up is performed by a multidisciplinary team comprised of a Maternal-Fetal-Medicine specialist, a hepatologist and a transplant surgeon. In our study, the first case series from Israel, we present our single-center experience managing pregnancies following liver transplantation during the past 4 years, and present maternal, fetal, neonatal and allograft outcomes. We also examined the effect of low-dose aspirin use on hypertensive disease incidence since the implementation of such treatment in pregnant livertransplant recipients at our institution beginning 2018. 


\section{Materials and Methods}

We performed an observational study examining 13 deliveries among 10 liver-transplant recipients, delivering between 2016 and 2020 at our medical center. Complete data for retrospective analysis were obtained from the Maternal-Fetal Medicine and Hepatology clinic hospital records. The following maternal and obstetric parameters before and during pregnancy were assessed: primary liver disease, age at liver transplantation, age at conception, time interval between transplantation and delivery, immunosuppressive regimen received, prepregnancy co-morbidities (diabetes mellitus and hypertension), gravidity, parity and aspirin use.

Obstetric complications were evaluated and included: Mode of delivery, preterm birth (defined as delivery before 37 weeks of gestation), gestational hypertension, pre-eclampsia, gestational diabetes mellitus, cholestasis, anemia, urinary tract infections and other infections, graft rejection, postpartum fever, postpartum hemorrhage, and blood transfusion.

Neonatal outcomes were assessed and included: gestational age at delivery, birthweight, Apgar score $<7, \mathrm{PH}<7.15$, neonatal death, jaundice requiring phototherapy, NICU admission, respiratory composite (respiratory distress syndrome (RDS), transient tachypnea of the newborn (TTN) or need for mechanical ventilation), and sepsis. 


\section{Results}

We identified 13 deliveries in 10 pregnant liver transplant recipients. The indications for liver transplantation included: acute liver failure (3 recipients), Wilson's disease (4 recipients), liver failure caused by Hepatitis A virus (1 recipient), primary sclerosing cholangitis (1 recipient) and heperxaluria type 1 ( 1 recipient) (Table 1). Median maternal age at liver transplant was 23 (range 4-33), median age at conception was 30 (range 25-35), and median time between organ transplantation and delivery was 4.5 years (range 0-26 years). 6 women were nulliparous, 2 had pre-gestational diabetes and 1 suffered from depression (Table 2).

All recipients were treated with tacrolimus in all pregnancies, and steroids were administered to 9 recipients (69\%). Low-dose aspirin (100 mg daily) was administered at our institution to pregnant liver-transplant recipients beginning 2018 and was given in 5 (38\%) of the pregnancies described here (Table 2).

\section{Obstetric and neonatal outcomes}

Regarding maternal outcomes (Table 3), 2 women (16\%) suffered from pre-eclampsia, 4 (32\%) from diabetes of any kind (pre-gestational diabetes mellitus or gestational diabetes), and 6 (46\%) delivered by cesarean section for various indications ( 3 due to abnormal lie, 1 due to non-reassuring fetal heart rate, 1 due to liver transplantation during pregnancy and one due to previous CS ). There were no cases of maternal death. Elevated liver enzymes were observed in 3 patients and resolved after delivery, with no cases of acute rejection identified.

Cholestasis occurred in 4 cases (32\%), thrombocytopenia in 4 (32\%) and infection (UTI, bacteremia, sepsis, endometritis) in 3 (24\%). Post-partum hemorrhage occurred in 2 cases $(16 \%)$, with one woman requiring blood products transfusion. There were no cases of placental abruption or admissions to the ICU during or after pregnancy.

Regarding neonatal outcomes (Table 4), median gestational age at delivery was 37 weeks (range, 31-39), with 6 (48\%) women experiencing preterm birth (range 31-36 weeks), with a median birthweight of $3072 \mathrm{~g}$ (range, 1450-4100 g). 5-min-apgar scores were all >8, PH was $>7.15$, and 2 neonates (16\%) were admitted to the NICU. No cases of sepsis, asphyxia or intrauterine fetal death were observed. 
None of the five women receiving low-dose aspirin beginning 12 weeks of gestation until 35 weeks, developed hypertensive disease or preeclampsia, while 2 of the 8 women not receiving aspirin (25\%) developed mild preeclampsia, and one (12.5\%) developed gestational hypertension. (Table 5). None of the aspirin treated patients suffered excessive bleeding during pregnancy.

\section{Two unusual pregnancies:}

Of note, one woman in our cohort underwent a liver transplantation in her 29th week of gestation because of acute liver failure attributed to propylthiouracil (PTU) use. She delivered by cesarean section at 31 weeks due to elevated liver enzymes and suspected intra-uterine growth restriction.

Another patient, a liver transplant recipient subsequent to Wilson's disease with pregestational diabetes and depression, presented at 32 weeks of gestation with sepsis and fever, and was delivered by cesarean section due to non-reassuring fetal heart rate and an abnormal biophysical profile. 


\section{Discussion}

In this summary of our single-center institutional experience, we demonstrated that pregnancy after liver transplantation holds good results for the mother, neonate and allograft. No maternal or fetal deaths occurred and there was no acute allograft rejection.

Despite the limited number of cases described in the literature, our results match larger case series outcomes, such as that depicted by Kubo et al (3), with the incidence of pre-term birth being $48 \%$, cesarean section rate $46 \%$, pre-eclampsia $16 \%$, and low birth weight $24 \%$. The immunosuppressive medication regimen in our cohort is in accordance with the treatment recommended in such cases in previous publications, with all patients receiving tacrolimus and most receiving steroids $(1,2,8)$.

Low-dose aspirin treatment for pregnant liver transplant recipients, with the aim of reducing the risk for preterm preeclampsia, began in 2018 at our institution after the results of the ASPRE study (5), and owing to the subsequent favourable results and effects we saw at our institution with such treatment in pregnant kidney transplant recipients $(4,5,7)$.

The incidence of preeclampsia in pregnant liver transplant recipients in our study was $16.6 \%$. No case of pre-eclampsia was seen in the aspirin-treated women, compared to a rate of $28 \%$ in the no-aspirin group (Table 5). Whilst previous studies have reported rates ranging between $21 \%-26 \%$ for pre-eclampsia in liver-transplanted pregnant women, more recent studies reported a lower incidence for pre-eclampsia ranging between $7 \%-12 \%$. $(1,3,6)$.

Preeclampsia is a form of hypertension unique to human pregnancy, and is considered a complex, multisystem heterogenous condition, that may originate from multiple causes. It is believed to evolve from changes in placental development that result in placental ischemia, thereby producing inflammation and oxidative stress. This stress may be a result of pathological placental development and placental ischemia, an overactive inflammatory response to normal placentation or due to a pre-existing inflammatory condition. Organ transplant recipients hold at least two of these risk factors - a higher risk for pathological placental development and a pre-existing inflammatory state (4).

The use of drugs with anti-inflammatory, anti-angiogenic, and antiplatelet properties, such as low-dose aspirin, holds a preventive effect on the risk for preeclampsia development. A recent 
review by Rahim et al (6) linked this preventive effect to increased management of immunosuppression and risk factors associated with pre-eclampsia. Rahim's team also recommended daily aspirin treatment starting before 16 weeks of gestation in order to improve placental hemodynamics and reduce the risk for preterm pre-eclampsia in women at risk.

Post-partum infection in liver transplant recipients is another pregnancy-related complication obstetricians need to be aware of. In our study, 2 women were diagnosed with sepsis due to urinary tract infection, and another patient was diagnosed with endometritis. This is higher than the rate in the general obstetric population, where 3\% suffer from UTI post-partum and 2\% from endometritis (9), most probably secondary to immunosuppression. Expeditious diagnosis and treatment can be lifesaving for these immunosuppressed women.

Our study has several limitations. First, the small number of cases included precludes reaching statistically significant conclusions regarding outcomes and management in this patient population, although due to the rarity of the entity of pregnant liver transplanted women, reaching a proper sample size for adequate power is challenging, especially at a single-center. Future studies combining multi-center experiences may shed light on this important and complex group of pregnant women. Secondly, another inherent limitation lies in the observational and retrospective nature of our study, requiring future validation in prospective studies, especially with regards to the use of low-dose aspirin in this patient population.

\section{Conclusion}

Liver transplanted pregnant women comprise a unique and complex patient group, requiring close surveillance by a multidisciplinary team. Relying on our single-center experience, we recommend considering low-dose aspirin treatment starting 12-16 weeks of pregnancy in liver transplant recipients, with the aim of preventing pre-eclampsia and its complications. There is need for further prospective studies regarding low-dose aspirin use in liver transplant recipients during pregnancy, in order to reduce complications and improve maternal and neonatal outcomes. 


\section{Declarations:}

All methods were carried out in accordance with relevant guidelines and regulations.

Informed consent: The study was approved by Rabin Medical Center's local Institutional Review Board (Study code 0339-19-RMC). All procedures performed in studies involving human participants were in accordance with the ethical standards of the institutional and/or national research committee and with the 1964 Helsinki declaration and its later amendments or comparable ethical standards. Informed consent was waived by Rabin Medical Center's local Institutional Review Board who approved the study, due to the study's retrospective design.

Consent to publication: Not applicable.

Data availability: All data generated or analysed during this study are included in this published article.

Disclosure of potential conflicts of interest: The authors have no relevant financial or nonfinancial interests to disclose.

Funding: None.

\section{Authors' contributions:}

- Gil Zeevi, MD - Substantial contributions to conception and design; acquisition of data; analysis and interpretation of data; drafting the article and revising it critically for important intellectual content; final approval of the version to be published.

- Marius Braun, MD - drafting the article and revising it critically for important intellectual content; final approval of the version to be published.

- Eviatar Nesher, MD - drafting the article and revising it critically for important intellectual content; final approval of the version to be published.

- Arnon Wiznitzer, MD - drafting the article and revising it critically for important intellectual content; final approval of the version to be published.

- Eran Hadar, MD - drafting the article and revising it critically for important intellectual content; final approval of the version to be published.

- Alyssa Hochberg, MD MPH - Substantial contributions to conception and design; analysis and interpretation of data; drafting the article and revising it critically for important intellectual content; final approval of the version to be published.

- Acknowledgements: Not applicable. 


\section{References}

1. Coffin CS, Shaheen AA, Burak KW, Myers RP. Pregnancy outcomes among liver transplant recipients in the United States: a nationwide case-control analysis. Liver transplantation. $2010 \mathrm{Jan}$; 16(1): 56-63.

2. Alisi A, Balsano C, Bernabucci V, Berzigotti A, Brunetto M, Bugianesi E, et al. AISF position paper on liver transplantation and pregnancy: Women in Hepatology Group, Italian Association for the Study of the liver (AISF). Digestive and liver disease. 2016; 48(8): 860-8.

3. Kubo S, Uemoto S, Furukawa H, Umeshita K, Tachibana D, Japanese Liver Transplantation Society. Pregnancy outcomes after living donor liver transplantation: results from a Japanese survey. Liver transplantation. 2014 May; 20(5): 576-83.

4. LeFevre ML. Low-dose aspirin use for the prevention of morbidity and mortality from preeclampsia: US Preventive Services Task Force recommendation statement. Annals of internal medicine. 2014 Dec 2; 161(11): 819-26.

5. Rolnik DL, Wright D, Poon LC, O'Gorman N, Syngelaki A, de Paco Matallana C, Akolekar R, Cicero S, Janga D, Singh M, Molina FS, Persico N, Jani JC, Plasencia W, Papaioannou G, Tenenbaum-Gavish K, Meiri H, Gizurarson S, Maclagan K, Nicolaides KH. Aspirin versus Placebo in Pregnancies at High Risk for Preterm Preeclampsia. N Engl J Med. 2017 Aug 17; 377(7): 613-622. doi:

6. Rahim MN, Long L, Penna L, Williamson C, Kametas NA, Nicolaides KH, Heneghan MA. Pregnancy in liver transplantation. Liver Transplantation. 2020 Apr; 26(4): 564-81

7. Coomarasamy A, Honest H, Papaioannou S, Gee H, Khan KS. Aspirin for prevention of preeclampsia in women with historical risk factors: a systematic review. Obstet Gynecol. 2003 Jun; 101(6): 1319-32. doi: 10.1016/s0029-7844(03)00169-8. PMID: 12798543.

8. Kanzaki Y, Kondoh E, Kawasaki K, Mogami H, Chigusa Y, Konishi I. Pregnancy outcomes in liver transplant recipients: A 15-year single-center experience. Journal of Obstetrics and Gynaecology Research. 2016 Nov; 42(11): 1476-82.

9. Axelsson D, Blomberg M. Prevalence of postpartum infections: a population-based observational study. Acta Obstet Gynecol Scand. 2014 Oct; 93(10): 1065-8. 


\section{Tables}

\begin{tabular}{|l|l|l|}
\hline \multicolumn{2}{|l|}{ Table 1 - Primary Liver Diseases } \\
\hline Primary Liver Disease & $\begin{array}{l}\text { Number of Women } \\
\mathrm{n}=10\end{array}$ & $\begin{array}{l}\text { Number of Pregnancies } \\
\mathrm{n}=13\end{array}$ \\
\hline Wilson & $4(40 \%)$ & $7(53 \%)$ \\
\hline Acute liver failure NOS & $3(30 \%)$ & $3(23 \%)$ \\
\hline HAV $^{\mathrm{b}}$ & $1(10 \%)$ & $1(8 \%)$ \\
\hline Hyperoxaluria type 1 & $1(10 \%)$ & $1(8 \%)$ \\
\hline PSC $^{\mathrm{c}}$ & $1(110 \%)$ & $1(8 \%)$ \\
\hline
\end{tabular}

Continuous variables are presented as median (range) and categorical numbers as $\mathrm{n}(\%)$.

${ }^{a} \mathrm{NOS}=$ Not otherwise specified

${ }^{\mathrm{b}} \mathrm{HAV}=$ Hepatitis A virus

${ }^{\mathrm{c} P S C}=$ Primary sclerosing cholangitis 
Table 2 - Characteristics of the 13 Pregnancies

\begin{tabular}{|l|l|}
\hline & Values \\
\hline Age at liver transplant (years) & $23(4-33)$ \\
\hline Age at birth (years) & $30(25-35)$ \\
\hline Transplant-to-delivery interval & \\
\hline & $2 \quad(1 \quad$ during \\
Less than 2 years & pregnancy) \\
\hline More than 2 years & 11 \\
\hline Gravidity & $1-5$ \\
\hline Parity & \\
\hline 0 & $5(38.5)$ \\
\hline 1 & $5(38.5)$ \\
\hline 2 & $3(23)$ \\
\hline DM & $2(17)$ \\
\hline HTN & \\
\hline BMIc & $0(0)$ \\
\hline Normal (18-25) & \\
\hline$>25$ & $10(77)$ \\
\hline$>30$ & $3(23)$ \\
\hline Immunosuppressive Medications & $13(100)$ \\
\hline Prograf & $9(69 \%)$ \\
\hline Prednisone & $5(38 \%)$ \\
\hline Aspirin & \\
\hline & \\
\hline & \\
\hline
\end{tabular}

Continuous variables are presented as median (range) and categorical numbers as $\mathrm{n}(\%)$.

${ }^{a} \mathrm{DM}=$ Diabetes Mellitus

${ }^{\mathrm{b}} \mathrm{HTN}=$ Hypertension

${ }^{\mathrm{c}} \mathrm{BMI}=$ Body mass index $\left(\mathrm{kg} / \mathrm{m}^{2}\right)$

${ }^{\mathrm{d}}$ Number of cases out of the 10 pregnant cases 


\begin{tabular}{|l|l|l|}
\hline \multicolumn{2}{|l|}{ Table 3 - Obstetric outcomes } \\
$n=13(\%)$ & \\
\hline $\begin{array}{l}\text { Mode of delivery } \\
\text { Normal vaginal delivery } \\
\text { Vacuum extraction delivery } \\
\text { Cesarean section }\end{array}$ & $\begin{array}{l}\text { 1(4) } \\
6(46)\end{array}$ & \\
\hline Preterm birth (<37weeks) & $6(46)$ & \\
\hline Gestational hypertension & $1(8)$ & \\
\hline Preeclampsia & $2(16)$ & \\
\hline Gestational diabetes & $2(16)$ & \\
\hline Thrombocytopenia & $4(32)$ & \\
\hline Cholestasis & $3(24)$ & \\
\hline Elevated liver enzymes & $3(24)$ & \\
\hline Postpartum infection & $3(24)$ & \\
\hline Postpartum hemorrhage & $2(16)$ & \\
\hline Postpartum infection & $3(24)$ & \\
\hline Severe anemia & 0 & \\
\hline Blood transfusion & $1(8)$ & \\
\hline Graft rejection & 0 & \\
\hline
\end{tabular}

Continuous variables are presented as median (range) and categorical numbers as n (\%). 
$\underline{\text { Table } 4 \text { - Neonatal outcomes }}$

\begin{tabular}{|c|c|}
\hline 5-minute Apgar score & \\
\hline Less than 7 & 0 \\
\hline More than 7 & 13 \\
\hline Gestational age at delivery & \\
\hline Less than 32 weeks & $1(8 \%)$ \\
\hline 32-37 weeks & $5(38 \%)$ \\
\hline More than 37 weeks & $7(54 \%)$ \\
\hline Birthweight & \\
\hline $1000-1500$ grams & $1(8 \%)$ \\
\hline $1500-2500$ grams & $2(16 \%)$ \\
\hline More 2500 grams & $10(72 \%)$ \\
\hline Congenital anomalies & 0 \\
\hline $\begin{array}{l}\text { Neonatal intensive care unit } \\
\text { (NICU) admission }\end{array}$ & $2(18 \%)$ \\
\hline $\mathrm{PH}<7.15$ & 0 \\
\hline Neonatal death & 0 \\
\hline $\begin{array}{l}\text { Jaundice requiring } \\
\text { phototherapy }\end{array}$ & $1(8 \%)$ \\
\hline Respiratory composite $^{a}$ & $1(8 \%)$ \\
\hline Blood transfusion & $1(8 \%)$ \\
\hline sepsis & 0 \\
\hline
\end{tabular}

Continuous variables are presented as median (range) and categorical numbers as $\mathrm{n}(\%)$.

${ }^{\text {a }}$ Respiratory composite $=$ respiratory distress syndrome (RDS), transient tachypnea of the newborn (TTN) or need for mechanical ventilation. 
Table 5-Aspirin treatment

\begin{tabular}{|l|l|l|l|}
\hline & Number of pregnancies & Pre-eclampsia & $\%$ \\
\hline No aspirin & 8 & 2 & $25 \%$ \\
\hline Aspirin & 5 & 0 & 0 \\
\hline
\end{tabular}

